\title{
Turbulent Heating in the Galactic Diffuse Ionized Gas
}

\author{
Anthony Minter and Dana S. Balser
}

National Radio Astronomy Observatory ${ }^{\star \star}$, Green Bank, West Virginia, U.S.A. 24944

\begin{abstract}
The observed properties of the diffuse ionized gas (DIG) in our Galaxy are not easily reconcilable with simple photoionization models. This suggests that there are different or additional physical processes at work in the DIG. We have developed a model of the DIG whereby it is ionized by a relatively soft ionizing spectrum $\left(T_{\text {eff }} \leq 32,000 \mathrm{~K}\right)$ and is also heated by an additional thermal mechanism: the dissipation of turbulence. This model predicts the same electron temperature, [ $\mathrm{N}$ II] $\lambda 6583 / \mathrm{H} \alpha$ ratio, [S $\mathrm{II}] \lambda 6716 / \mathrm{H} \alpha$ ratio and $\mathrm{He}$ I $\lambda$ 5876/ $\mathrm{H} \alpha$ ratio as observed in the DIG. Without the turbulent thermal heating term, this model will not reproduce the observed properties of the DIG. The dissipation of turbulence may also be important in other phases of the ISM.
\end{abstract}

\section{Modeling the Diffuse Ionized Gas}

The diffuse ionized gas (DIG), also referred to as the Reynolds layer, is a nearly fully ionized gas with a mean midplane density of $0.025 \mathrm{~cm}^{-3}$ and a scale height of $\sim 1 \mathrm{kpc}$. The energy required to keep the DIG ionized is $\geq$ $10^{-4} \mathrm{ergs} \mathrm{cm}^{-3} \mathrm{~s}^{-1}$, corresponding to $5 \times 10^{6}$ hydrogen ionizing photons per $\mathrm{cm}^{-2}$ per sec in the Galactic disk (Reynolds 1992). The He I $\lambda$ 5876/H $\alpha$ ratio indicates that the ionizing spectrum incident on the DIG is relatively soft and corresponds to a helium to hydrogen ionization factor of $\chi_{\mathrm{He}} / \chi_{\mathrm{H}} \lesssim 0.5$ (Tufte 1997). The observed ranges of $[\mathrm{N} \mathrm{II}]$ and $[\mathrm{S} \mathrm{II}]$ relative to $\mathrm{H} \alpha$ require a much harder ionizing spectrum if photoionization is to explain the line ratios (Reynolds 1985a). Previous models (e.g., Domgörgen \& Mathis 1994) have attempted to explain the conditions in the DIG but have not been entirely successful (Reynolds \& Tufte 1995; Rand 1997).

In classical turbulence theory, the energy contained in the large scale "eddies" cascades to smaller scale eddies. This cascade of energy to smaller scales occurs on all spatial scales down to a dissipative scale, at which point the turbulent energy is converted into thermal energy via some dissipation mechanism. The dissipation rate of the turbulence is proportional to the energy contained in the turbulence (Minter \& Spangler 1996). Minter \& Spangler

* The National Radio Astronomy Observatory is a facility of the National Science Foundation operated under cooperative agreement by Associated Universities, Inc. 
Table 1. Observed Properties of the DIG

\begin{tabular}{|c|c|c|}
\hline Quantity & |Observed Range| & Reference \\
\hline$T_{\mathrm{e}}$ & $8,000 \pm 2,000 \mathrm{~K} \mid$ & Reynolds 1989, Reynolds 1985a \\
\hline He I $\lambda 5876 / \mathrm{H} \alpha$ & $\lesssim 0.02$ & Reynolds \& Tufte 1995, Tufte 1997 \\
\hline$[\mathrm{N}$ II $] \lambda 6583 / \mathrm{H} \alpha$ & $0.2-0.5$ & Reynolds 1985a \\
\hline$[\mathrm{S}$ II $] \lambda 6716 / \mathrm{H} \alpha$ & $0.2-0.5$ & Ogen \& Reynolds 1985, Reynolds et al. 1977 \\
\hline$[\mathrm{O}$ I $] \lambda 6300 / \mathrm{H} \alpha$ & $\leq 0.04$ & Reynolds 1990, Tufte 1997 \\
\hline$\chi_{\mathbf{H}}$ & $90-97 \%$ & Tufte 1997 \\
\hline
\end{tabular}

(1996) determined the turbulent energy density in the DIG. From this information, Minter \& Spangler (1997) determined that the turbulent dissipation rate, and thus the turbulent heating rate, is likely to be in the range $10^{-25}-10^{-27} \mathrm{ergs} \mathrm{cm}^{-3} \mathrm{~s}^{-1}$ for the DIG, assuming $\chi_{\mathrm{H}}>0.9$. This value is approximately equal to the cooling rate of the DIG, indicating that the heating of the DIG via the dissipation of turbulence could be an important mechanism. The turbulent heating rate in $\mathrm{H}$ II regions is of the order of $10^{-21}-10^{-24} \mathrm{ergs} \mathrm{cm}^{-3} \mathrm{~s}^{-1}$ which is at least two orders of magnitude less than the cooling rate (Minter \& Balser 1997). Therefore, heating via the dissipation of turbulence is not a significant process in determining the observed line emissions of $\mathrm{H}$ II regions whereas it may be for the DIG and other phases of the ISM.

The numerical program CLOUDY (version 90.02) is used to model the diffuse ionized gas (DIG) near the Galactic midplane (see Ferland 1996 for a complete description of CLOUDY). The interstellar medium is composed of several different phases. We use CLOUDY to model only the diffuse ionized component which we assume is physically distinct, i.e. the DIG is composed of nearly fully ionized hydrogen. Observations of the DIG are used to constrain the physical parameters defined in CLOUDY (see Minter \& Balser 1997 for a detailed discussion of how CLOUDY was used).

\section{Results and Conclusions}

Figure 1 summarizes the models for a range of effective stellar temperatures $\left(T_{\text {eff }}\right)$. Notice that none of the models with only photoionization heating (dashed lines) satisfy the observational constraints. When the turbulent heating term is included (solid lines) there are many effective temperatures which satisfy all of the observational constraints. The He observations constrain the radiation field to be softer than $Q(\mathrm{He}) / Q(\mathrm{H}) \sim 5.2 \times 10^{-2}\left(T_{\text {eff }} \leq 32,000 \mathrm{~K}\right)$ for all models. The $[\mathrm{N} \mathrm{II}] \lambda 6583 / \mathrm{H} \alpha$ and $[\mathrm{S} \mathrm{II}] \lambda 6716 / \mathrm{H} \alpha$ ratios predicted 

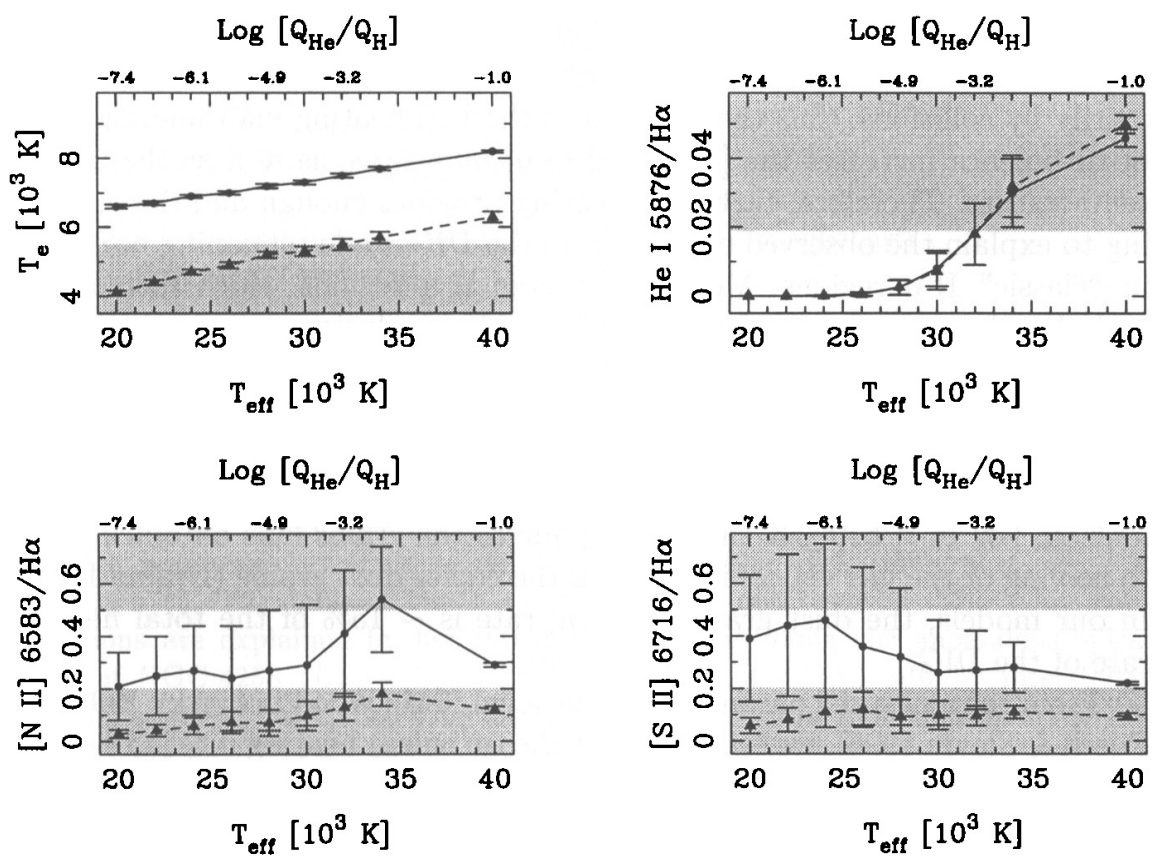

Fig. 1. Model results of the diffuse ionized gas (DIG). Plotted are the average values, over approximately the first $100 \mathrm{pc}$ out of the plane, of the electron temperature $\left(T_{\mathrm{e}}\right)$, the $\mathrm{He}$ I $\lambda 5876 / \mathrm{H} \alpha$ ratio, the $\left[\mathrm{N}_{\mathrm{II}}\right] \lambda 6583 / \mathrm{H} \alpha$ ratio, and the $[\mathrm{S}$ II] $\lambda 6716 / \mathrm{H} \alpha$ ratio as a function of the stellar effective temperature $\left(T_{\text {eff }}\right)$. The hardness of the ionizing spectrum is also described by $Q(\mathrm{He}) / Q(\mathrm{H})$ which is shown at the top of each figure. N.B., the effective temperature only determines the shape of the incident continuum and not the overall intensity. Each panel has two curves: the solid line (filled circles) includes the additional turbulent heating term, while the dashed line (filled triangles) excludes this term. The vertical lines correspond to the $1 \sigma$ variation in the mean values. Regions which are excluded observationally have been shaded gray (see Table 1).

by the models are consistent with the observations in Table 1 only when the turbulent heating is included.

All models predict that the $[\mathrm{O} \mathrm{III}] \lambda 5007 / \mathrm{H} \alpha$ ratio is $<0.005$, while Reynolds (1985b) measured an [O III] $\lambda 5007 / \mathrm{H} \alpha$ ratio of 0.06 in the diffuse ISM. The origin of this [O III] emission is not known. Our models suggest that the observed [O III] emission does not originate from the oxygen in the DIG. Our models also predict that the $\left[\mathrm{O}_{\mathrm{II}}\right] \lambda 3729 / \mathrm{H} \alpha$ line ratio ranges from 0.15 at $T_{\mathrm{e}}=20,000 \mathrm{~K}$ to 0.85 at $T_{\mathrm{e}}=40,000 \mathrm{~K}$. This ratio is at least five times greater than the values predicted by the models without turbulent heating.

A simple physical picture of the DIG evolves naturally from these results. The $\mathrm{H} \alpha$ and $\mathrm{He}$ I recombination lines are produced almost entirely by the 
stellar radiation field because the turbulent heating does not produce enough energy to ionize these species. The forbidden lines, however, are excited primarily by collisions; thus the addition of thermal heating via the dissipation of turbulence increases the $[\mathrm{N} \mathrm{II}]$ and $[\mathrm{S} \mathrm{II}]$ intensities as well as the kinetic temperature. Therefore, turbulent heating provides enough additional heating to explain the observed conditions in the DIG while remaining negligible in "classic" $\mathrm{H}$ II regions. Models of classic $\mathrm{H}$ II regions which include the turbulent heating discussed in $\$ 2$ confirm this conclusion.

Another heating mechanism which could be important in the DIG is photoelectric heating from dust grains (Reynolds \& Cox 1992, Draine 1978). This mechanism provides approximately the same heating rate as does the turbulent heating up to $T_{\mathrm{e}} \sim 6,000 \mathrm{~K}$ in a gas where the hydrogen is fully ionized. For $T_{\mathrm{e}} \geq 6,000 \mathrm{~K}$ the heating due to the grains falls off quickly due to cooling of the gas via collisions with the cooler dust grains (Draine 1978). In our models, the dust grains' heating rate is $\sim 15 \%$ of the total heating rate of the DIG.

From the measured parameters of the local ISM (Wood \& Linsky 1997 and Frisch \& Slavin 1996), we estimate that the turbulent heating rate is $10-50 \%$ of the cooling rate in the local interstellar cloud (LIC). The photoelectric heating from dust grains should also be important in the LIC (Frisch \& Slavin 1996). Thus the dissipation of turbulence should be important in the local ISM and may also be important in other phases of the ISM.

\section{References}

Domgörgen, H. \& Mathis, J.S., 1994, ApJ428, 647

Draine, B.T., 1978, ApJS36, 595

Ferland, G. J. 1996, Hazy, A Brief Introduction to Cloudy, University of Kentucky Department of Physics and Astronomy Internal Report

Frisch, P.C. \& Slavin, J.D., 1996, Space Science Reviews 78, 223 (Kluwer Academic Publishers)

Minter, A.H. \& Balser, D.S., 1997, ApJ484, in press

Minter, A.H. \& Spangler, S.R., 1996, ApJ458, 194

Minter, A.H. \& Spangler, S.R., 1997, ApJ485, in press

Ogden, P. M., \& Reynolds, R.J. 1985, ApJ290, 238

Rand, R.J., 1997, ApJ474, 129

Reynolds, R.J., 1985a, ApJ294, 256

Reynolds, R.J., 1985b, ApJL298, L27

Reynolds, R.J., 1989, ApJ345, 811

Reynolds, R. J. 1990, ApJ 349, L17

Reynolds, R.J., 1992, ApJL392, L95

Reynolds, R.J. \& Cox, D.P., 1992, ApJL400, L33

Reynolds, R.J., Roesler, F. L., \& Scherb, F. 1977, ApJ211, 115

Reynolds, R.J. \& Tufte, S.L., 1995, ApJL439, L17

Tufte, S.L., Ph.D. thesis, University of Wisconsin-Madison

Wood, B. E. \& Linsky, J. L. 1997,ApJL474, L39 